\title{
Progression of physical inactivity in COPD patients: the effect of time and climate conditions - a multicenter prospective cohort study
}

This article was published in the following Dove Press journal: International Journal of Chronic Obstructive Pulmonary Disease

\author{
Afroditi K Boutou, (iD) 1,2,* Yogini \\ Raste, '* Heleen Demeyer, ${ }^{3}$ Thierry \\ Troosters, ${ }^{3}$ Michael I Polkey, (iD) ' \\ loannis Vogiatzis, (iD) ${ }^{4,5}$ Zafeiris \\ Louvaris, ${ }^{4}$ Roberto A Rabinovich, ${ }^{6}$ \\ Thys van der Molen, ${ }^{7}$ Judith \\ Garcia-Aymerich, (ID) ${ }^{8-10}$ Nicholas S \\ Hopkinson (iD)'
}

On behalf of the PROactive consortium

'National Institute for Health Research Respiratory Biomedical Research Unit, Royal Brompton and Harefield NHS Foundation Trust and Imperial College, London, UK;

${ }^{2}$ Department of Respiratory Medicine, "G. Papanikolaou” Hospital, Thessaloniki, Greece; ${ }^{3}$ Department of Rehabilitation Sciences, Faculty of Kinesiology and Rehabilitation Sciences and Respiratory Division, University Hospital Leuven, Leuven, Belgium; ${ }^{4}$ First Department of Respiratory Medicine, National \& Kapodistrian University of Athens, Athens, Greece; ${ }^{5}$ Department of Sport, Exercise and Rehabilitation, Northumbria University, Newcastle, UK; ${ }^{6}$ ELEGI Colt Laboratory, Centre for Inflammation Research, The Queen's Medical Research Institute, University of Edinburgh, Edinburgh, UK; ; Department of General Practice, University Medical Center

Groningen, University of Groningen, Groningen, The Netherlands; ${ }^{8}$ ISGlobal, Barcelona, Spain; ${ }^{9}$ Centro de Investigación Biomédica en Red de Epidemiología y Salud Pública (CIBERESP), Barcelona, Spain;

${ }^{10}$ Universitat Pompeu Fabra (UPF), Barcelona, Spain

*These authors contributed equally to this work

Correspondence: Afroditi K Boutou Department of Respiratory Medicine, "G. Papanikolaou" Hospital, Exohi, Thessaloniki 57010, Greece Tel +30 23I 3307277

Email afboutou@yahoo.com
Purpose: Longitudinal data on the effect of time and environmental conditions on physical activity (PA) among COPD patients are currently scarce, but this is an important factor in the design of trials to test interventions that might impact on it. Thus, we aimed to assess the effect of time and climate conditions (temperature, day length and rainfall) on progression of PA in a cohort of COPD patients.

Patients and methods: This is a prospective, multicenter, cohort study undertaken as part of the EU/IMI PROactive project, in which we assessed 236 COPD patients simultaneously wearing two activity monitors (Dynaport MiniMod and Actigraph GT3X). A multivariable generalized linear model analysis was conducted to describe the effect of the explanatory variables on PA measures, over three time points (baseline, 6 and 12 months).

Results: At 12 months ( $\mathrm{n}=157 ; \mathrm{FEV}_{1} \%$ predicted=57.7 \pm 21.9$)$ there was a significant reduction in all PA measures (Actigraph step count (4284 \pm 3533 vs $3533 \pm 293)$ ), Actigraph moderate- to vigorous-intensity PA ratio (8.8 (18.8) vs 6.1 (15.7)), Actigraph vector magnitude units $(374,902.4(265,269)$ vs 336,240 (214,432)), MiniMod walking time (59.1 (34.9) vs 56.9 (38.7) mins) and MiniMod PA intensity (0.183 (0) vs 0.181 (0)). Time had a significant, negative effect on most PA measures in multivariable analysis, after correcting for climate factors, study center, age, $\mathrm{FEV}_{1} \%$ predicted, $6 \mathrm{MWD}$ and other disease severity measures. Rainfall was the only climate factor with a negative effect on most PA parameters. Conclusion: COPD patients demonstrate a significant decrease in PA over 1 year follow-up, which is further affected by hours of rainfall, but not by other climate considerations.

Keywords: physical activity, chronic obstructive pulmonary disease, elapsed time, climate

\section{Introduction}

COPD is a chronic, debilitating disorder. ${ }^{1}$ Although progressive airflow limitation is the diagnostic feature of this disease, several other manifestations, such as exertional dyspnea, reduced exercise capacity, muscle weakness, reduced physical activity (PA) and poor health-related quality of life, are of major clinical importance for the COPD patient and may also worsen with time. ${ }^{2}$ Reduced daily PA is observed early in the course of the disease, ${ }^{3,4}$ has a range of causes and consequences, including an association with several systemic components of $\operatorname{COPD}^{5,6}$ and is a strong predictor of exacerbation rate, hospital admission and mortality. ${ }^{7}$ However, published data on longitudinal studies which evaluated changes in PA with time relative to other features of the disease are currently limited ${ }^{8-11}$ and are not without limitations. In a single-center study Waschki et al established that PA 
declines with time, ${ }^{9}$ evaluating a mixed population of COPD and chronic bronchitis patients, while in another longitudinal study with similar results, more than $90 \%$ of participants were male. ${ }^{11}$ Moreover, most published studies assessed changes in PA after a relatively long followup period of more than 2 years, ${ }^{9-11}$ during which several other disease features had also worsened.

Previous data indicate that weather conditions may be an important factor to consider when assessing the variability of daily PA. In a large systematic review looking at approximately 300,000 healthy individuals (adults, adolescents and children) from eight different countries, PA varied with seasonality and was found to be higher in the summer months and significantly lower during the winter. ${ }^{12}$ However, patterns of PA are not identical in health and disease, especially when exercise induces disease-specific symptoms. ${ }^{13}$ Currently, evidence of the specific effect of environmental conditions on PA among COPD patients is limited. ${ }^{14-17}$ In these studies, season and temperature were established as the most significant climate predictors of daily step count. Nevertheless, the relatively small patient cohort, ${ }^{12}$ wide variations in the follow-up period ${ }^{14}$ and the lack of objective indices to define seasons ${ }^{15,16}$ are some of the factors which limit the interpretation of these results. Moreover, all these studies ${ }^{14-16}$ were single-center studies, and therefore the important question of the effect of geographical area on PA variation among COPD patients living in different countries is currently unknown.

Against this background we conducted an analysis of data obtained during a prospective, multicenter, longitudinal study, undertaken as part of the EU/IMI PROactive project, ${ }^{18}$ in order to assess the change in PA levels over time, defined by three time point measurements (at baseline, 6 and 12 months), corrected for the potential effect of climate variation (as defined by weekly average of day length, temperature and rainfall) among COPD patients. We hypothesized that PA would decline with time, being lower at the end of the 12-month study period and that it would be affected by climate, being lower in conditions of cooler temperature, increased rainfall and shorter day length. This is important both for understanding the natural history of COPD and particularly for trial design when considering the size, setting and duration of studies with PA as an outcome measure, especially in trials with a multicenter protocol.

\section{Materials and methods}

\section{Study population}

The study population was recruited from five European centers as part of the PROactive study (Royal Brompton Hospital, London UK: ELEGI Colt Institute, Edinburgh, UK; KU Leuven, Belgium; First Department of Respiratory Medicine, National \& Kapodistrian University of Athens Medical School, Greece; Department of General Practice, University Medical Center Groningen, Netherlands). ${ }^{18}$ All had a clinical history compatible with COPD, spirometric evidence of chronic airflow limitation (post bronchodilation $\mathrm{FEV}_{1}$ to FVC ratio $<0.7$ ), and were clinically stable (have not used systemic antibiotics, systemic corticosteroids or being hospitalized) for a minimum of 4 weeks prior to entering the study. Patients who had co-morbidities which would interfere with their movement (e.g. orthopedic disorders or neuromuscular disease), respiratory diseases other than COPD (e.g. asthma), cognitive impairment, were excluded. All patients gave written informed consent for participation in the study, and the study protocol received ethical approval from the respective research ethics committees (Lothian Regional Ethics Committee, UK; UZ Leuven Medical Ethics Committee, Belgium; University Hospital Ethics Committee "Sotiria" Chest General Hospital Athens, Greece, University Medical Center Ethics Committee University of Groningen, The Netherlands) as well as local site-specific approval. We also confirm that this study was conducted in accordance with the Declaration of Helsinki. Some data from this cohort have been previously published. ${ }^{19,20}$ Application for access to deidentified patient data can be made to the PROactive consortium via the authors.

\section{Study protocol and measurements Study design}

This was a prospective cohort study with measurements at baseline, 6 and 12 months follow-up. Details of the study protocol and assessments are available on: www.clinical trials.gov (NCT01388218). ${ }^{18}$ Baseline measures included anthropometrics, spirometry, plethysmographic lung volumes, quadriceps maximal voluntary contraction (QMVC), COPD Assessment Test (CAT), ${ }^{21}$ Hospital Anxiety and Depression scale (HAD), ${ }^{22}$ Chronic COPD Questionnaire (CCQ), ${ }^{23}$ as well as $6 \mathrm{MWD}$ assessed according to guidelines, ${ }^{24}$ which included a practice walk.

\section{PA monitoring}

Patients were required to wear simultaneously two PA monitors previously validated in people with $\mathrm{COPD},{ }^{25,26}$ 
the Dynaport MiniMod (McRoberts BV, the Hague, the Netherlands) and Actigraph GT3X (Actigraph, Pensacola, FL, USA). ${ }^{26}$ Both monitors are trunk-worn triaxial accelerometers; the MiniMod was worn in the small of the back; the Actigraph was worn at the right hip.

Baseline assessment involved 2 consecutive weeks of PA monitoring. Patients were instructed not to wear the monitors at night when asleep or while bathing or swimming. Follow-up was conducted at 6 and 12 months from baseline, but with only 1 week of activity monitoring; this shorter period of monitoring was decided as a result of work previously published by the PROactive consortium. ${ }^{25}$ The measures of PA recorded by the Actigraph were step count, moderate-intensity to vigorous-intensity PA ratio (mvpa) and vector magnitude units (VMU) (that is the vectorial sum of activity counts in three orthogonal directions) per day, while the MiniMod recorded step count, time spend in walking (walktime), PA intensity (measured in metabolic equivalents) and VMU per day.

\section{Weather data}

Data specific to the time periods during which patients were studied, as well as to their location, were provided by the UK Meteorological Office (https://www.metoffice. gov.uk). The Meteorological Office provided weather information for all European centers. These data comprised daily highest temperature and information on number of hours of sunlight and hours of rainfall (any hour in which there was any rainfall at all was counted), per day. Daily weather data were available for every day of activity monitoring collected. Unfortunately, data on daily hours of sunshine were not available for two of the centers (Leuven and Groningen) so these centers could not be included in that analysis.

\section{Statistical analysis}

The following guidelines were used when processing the PA data: 1) at least $10 \mathrm{hrs}$ of activity data for a day were required for that day to be included in the analysis, 2) patients who did not participate in all three time points (baseline, 6 and 12 months) were excluded from final analysis and 3) data were analyzed on a patient and not on a day-by-day basis, for each time point (that is we summarized data on PA measurements and on climate variable measurements, for each study period). All analyses were carried out using IBM SPSS Version 19 for Windows XP. Continuous variables are presented as mean value $\pm 1 \mathrm{SD}$ or as median value (interquartile range) depending on the normality or not of their distribution, while categorical variables are presented as absolute and \% value. Normality of distribution of values was assessed using the Shapiro-Wilk normality test, while Q-Q plots were used for visual confirmation of distribution of data. One-way ANOVA or Kruskal-Wallis test was applied to compare normally or non-normally distributed quantitative variables across different geographical sites at baseline. Values of PA measures and 6MWD were also compared between baseline, 6 and 12 months using: 1) the repeated measures ANOVA for normally distributed variables; equality of the variances of differences was tested utilizing the Mauchly's test of sphericity and the Huynh-Feldt correction was used when assumption of sphericity was violated, while the Bonferroni post-hoc test was applied for pairwise comparisons between groups, and 2) the Friedman test for non-normally distributed variables. The pairwise comparisons were further conducted using the Wilcoxon rank test and the Bonferroni correction for multiple comparisons. The bivariate association between climate variables and each PA measure was plotted utilizing generalized additive models; temperature was linearly associated with all PA measures, while for rainfall and day length, appropriate cutoff points were determined based on the curves of the spline lines. Further analysis of results was set out a priori as follows: the null hypothesis was that there is no difference in PA measures between baseline, 6 and 12 months follow-up, when corrected for age at baseline, climate conditions, study site, lung function and 6MWD. To test this hypothesis, a generalized linear model analysis was applied with each PA measure as the dependent (response) variable. $^{26}$ The constructed models tested visit, study site, baseline measurements (age, $\mathrm{FEV}_{1} \%$ predicted, residual volume (RV) to total lung capacity (TLC) ratio\% predicted, 6MWD, QMVC) and climate variables of each time point (temperature, day length, rainfall) as potential predictors (explanatory variables), simultaneously. ${ }^{27}$ The Akaike's Information Criterion and the -2 Log-Likelihood function were used to select the best model fit. ${ }^{28}$ The gamma distribution was chosen to fit PA measures distribution, while the link function which was finally selected was Log, so the $\mu$ i of each response variable could be expressed as: $\mu \mathrm{i}=\mathrm{g}-1(\eta \mathrm{i})=\mathrm{e}^{\eta \mathrm{i}}$. A level of $p<0.05$ was considered significant for all analyses.

\section{Results Study population}

A total of 236 COPD patients $(67.4 \pm 8.4$ years old; $57 \pm 20.5 \% \mathrm{FEV}_{1} \%$ predicted; $67.8 \%$ male) were recruited from five different European centers. COPD patients 
represented a wide range of disease severity: 15\% $\left(n_{1}=34\right)$ were GOLD stage I, 46\% $\left(\mathrm{n}_{2}=108\right)$ were GOLD stage II, $30 \%\left(n_{3}=71\right)$ were GOLD stage III and $9 \%\left(n_{4}=22\right)$ were GOLD stage IV. The baseline population characteristics and the baseline climate conditions, along with a breakdown by center are presented in online Table S1. However, not all patients completed the study (Figure 1). Table 1 presents baseline characteristics of the final study population, that is of the 157 patients (67.2 \pm 7.8 years old; $\mathrm{FEV}_{1}=57.7 \pm 21.9 \%$ predicted; $75.8 \%$ male) who finally completed both the 6 and the 12 months follow-up and were, thus, included in further analysis. Several baseline differences were noted between centers. For example, patients in Athens, who were recruited from a hospital outpatient clinic, had lower $\mathrm{FEV}_{1} \%$ predicted, QMVC and 6MWD, compared to patients in most of the other centers. Patients in Edinburgh, on the other hand, were older and had higher QMVC, compared to the rest. Patients in Leuven had higher baseline step counts and other PA measures, while in Groningen, where a larger proportion of patients came from primary care, patients were younger and presented with higher 6MWD, compared to other centers. Baseline climate conditions were also significantly different between the centers, with longer day length, higher temperature, more sunshine hours and much less hours with rainfall in Athens than the rest (Table 1). Overall, gender ratio, body mass index (BMI), RV/TLC\% predicted, HAD anxiety (A), HAD depression (D), CAT and CCQ scores were the only baseline variables found to be similar across all study sites (Table 1).

\section{Changes in PA levels with time}

Univariate analysis indicated that PA declined with time. All PA measures, apart from PA intensity, were significantly higher at baseline, compared both to 6 months and to 12 months follow-up (Table 2). For instance, the median number of steps had dropped by approximately

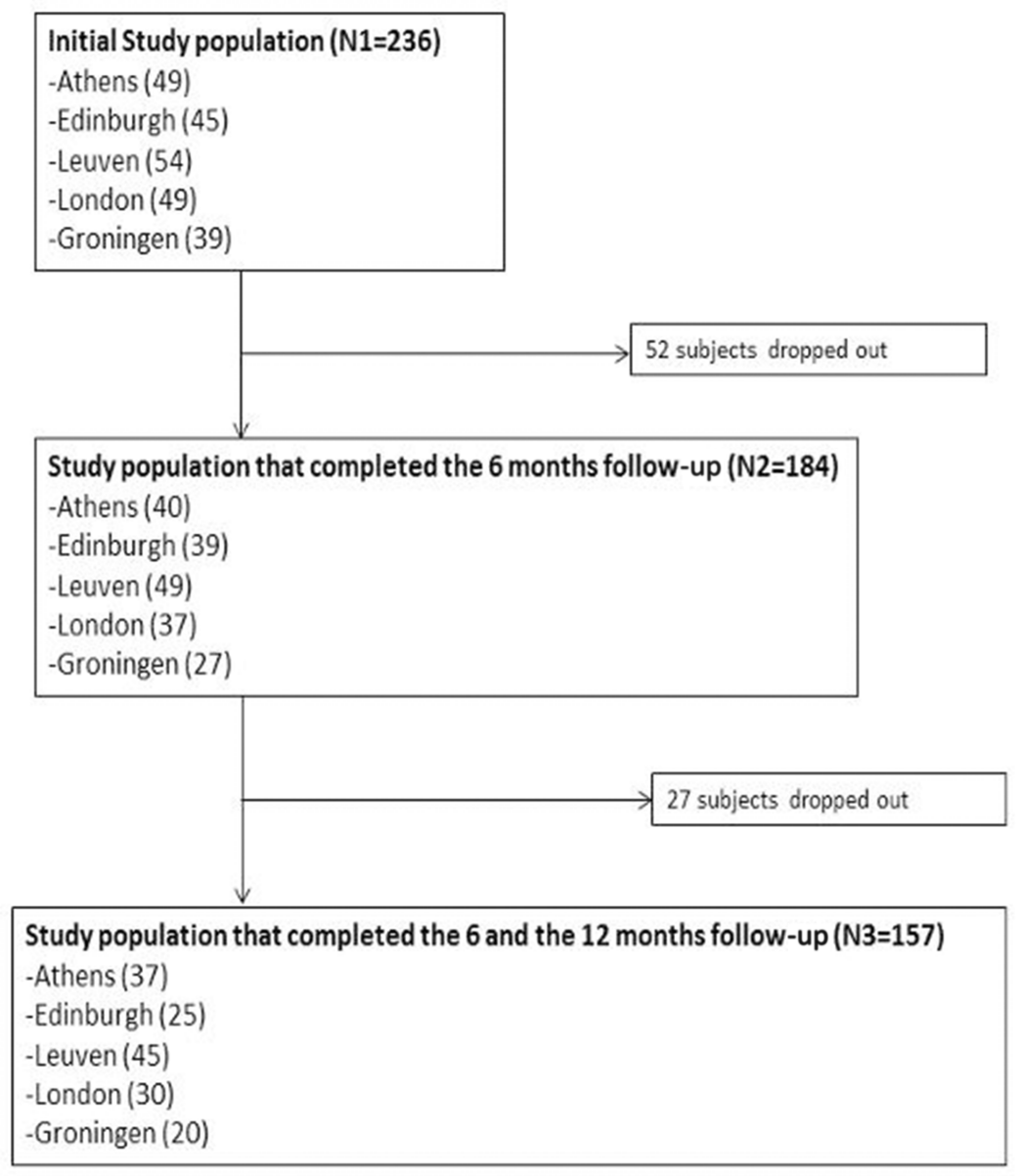

Figure I Study flowchart. 
Table I Baseline characteristics of patients who completed follow up and separated by center

\begin{tabular}{|c|c|c|c|c|c|c|c|}
\hline Variable & $\begin{array}{l}\text { Total population } \\
(\mathrm{N}=\mid \mathbf{5 7})\end{array}$ & $\begin{array}{l}\text { Athens } \\
(\mathrm{N} I=37)\end{array}$ & $\begin{array}{l}\text { Edinburgh } \\
(\mathrm{N} 2=25)\end{array}$ & $\begin{array}{l}\text { Leuven } \\
(\mathrm{N} 3=45)\end{array}$ & $\begin{array}{l}\text { London } \\
(\mathbf{N} 4=30)\end{array}$ & $\begin{array}{l}\text { Groningen } \\
\text { (N5=20) }\end{array}$ & $p$-value \\
\hline Recruitment site & $\begin{array}{l}\text { Hospital/rehabilitation/ } \\
\text { primary care }\end{array}$ & Hospital & Hospital & Rehabilitation & Hospital & Primary care & NA \\
\hline Age (years) & $67.2 \pm 7.8$ & $66.2 \pm 8.3$ & $7 I \pm 7.1$ & $66.4 \pm 6.3$ & $68.8 \pm 8.4$ & $63.8 \pm 8.9$ & 0.019 \\
\hline Male/female & $119: 38$ & $27: 10$ & $20: 5$ & $36: 9$ & $21: 9$ & $15: 5$ & 0.846 \\
\hline BMI $\left(\mathrm{kg} / \mathrm{m}^{2}\right)$ & $26.7 \pm 4.9$ & $26.7 \pm 6.1$ & $27.3 \pm 4.3$ & $27.1 \pm 4.7$ & $25.5 \pm 5$ & $26.8 \pm 3.3$ & 0.684 \\
\hline $\mathrm{FEV}_{1} \%$ & $57.7 \pm 21.9$ & $48.3 \pm 15.5$ & $61.6 \pm 24.2$ & $64.6 \pm 22$ & $54 \pm 25.2$ & $60.1 \pm 18.4$ & 0.009 \\
\hline $\mathrm{RV} / \mathrm{TLC} \%$ & $50.2 \pm 11.9$ & $50.9 \pm 13.6$ & $47.9 \pm 9.5$ & $50.3 \pm 13$ & $52.5 \pm 9.3$ & $47.8 \pm 11.7$ & 0.576 \\
\hline 6MWD (m) & $449.1 \pm 124.1$ & $381.5 \pm 73.2$ & $424.6 \pm 119.2$ & $502 \pm 142.7$ & $440.5 \pm 120.3$ & $497.7 \pm 100.6$ & $<0.001$ \\
\hline QMVC (kg) & $33.4 \pm 32.4$ & $27.9 \pm 7.8$ & $4 I \pm I I .1$ & $35.1 \pm 10$ & $30.6 \pm 9.5$ & $34.2 \pm 15.9$ & $<0.001$ \\
\hline AG steps & $4284(3533)$ & 3807 (3585) & $3338(2615)$ & $5166(4216)$ & 4207 (3552) & $3784(3710)$ & 0.238 \\
\hline AG mvpa & $8.8(18.8)$ & $7.8(18.8)$ & $7.2(11.4)$ & $9.9(20.6)$ & I2.1 (29.3) & $7.5(10.7)$ & 0.663 \\
\hline \multirow[t]{2}{*}{ AG VMU } & 374902.4 (265269) & 309289.1 & 322229.6 & 453780 & 356768.9 & 385016.8 & 0.178 \\
\hline & & (194928) & $(161516)$ & $(238837)$ & $(284389)$ & $(228490)$ & \\
\hline MM steps & $4690(3708)$ & $4135(3486)$ & $4070(2554)$ & $5774(44 \mid 8)$ & $3583(3600)$ & $4733(423 I)$ & 0.005 \\
\hline MM walktime (mins) & $59.1(34.9)$ & $54.3(40.7)$ & $52(19.2)$ & $75.6(42)$ & $48(43.5)$ & $57.1(47.7)$ & 0.003 \\
\hline MM intensity (g) & $0.2(0)$ & $0.2(0)$ & $0.2(0)$ & $0.2(0)$ & $0.2(0)$ & $0.2(0)$ & 0.080 \\
\hline \multirow[t]{2}{*}{ MM VMU } & $286039.6(237721)$ & 234813.2 & 215748 & 364976.9 & 297922.1 & 332468.7 & 0.057 \\
\hline & & $(245045)$ & $(122400)$ & $(33 \mid 440)$ & $(230476)$ & $(27008 I)$ & \\
\hline Temp $\left({ }^{\circ} \mathrm{C}\right)$ & I7.| (7.5) & $20.7(14)$ & $14.9(4.8)$ & I6.I (6.9) & $19.4(7.2)$ & $12.9(5)$ & $<0.001$ \\
\hline Sunshine (hrs) & $4.7(3.5)$ & $6.5(4)$ & $2.6(1.5)$ & - & $4.3(2.7)$ & - & $<0.001$ \\
\hline Rainfall (hrs/day) & $0.2(1.2)$ & $0(0)$ & I.4 (I.9) & $0.3(1.3)$ & $0.3(I)$ & $0.1(0.9)$ & $<0.001$ \\
\hline Day length (mins) & $640.2(158.4)$ & $662.3(117.5)$ & $647.2(191.6)$ & $647.4(259)$ & $694.1(211)$ & $589.3(80.8)$ & $<0.001$ \\
\hline $\operatorname{HAD} A^{\$}$ & $5(7)$ & $6(7)$ & $6(4.5)$ & $4.5(6.8)$ & $5(6.3)$ & $3(4.8)$ & 0.265 \\
\hline HAD D $\$$ & $4(5)$ & $5(4.5)$ & $35(4.5)$ & $4(5.8)$ & $4(4.5)$ & $2.5(5.5)$ & 0.176 \\
\hline$C A T^{\$}$ & $13(10.5)$ & $14(8.5)$ & $12(9)$ & $14(18)$ & $11(16)$ & $10.5(9.5)$ & 0.832 \\
\hline $\mathrm{CCQ}^{\$}$ & $1.7(1.6)$ & $1.6(1.4)$ & $1.6(1.6)$ & $1.4(\mathrm{I} .2)$ & $2.1(2.2)$ & $1.2(1.9)$ & 0.473 \\
\hline
\end{tabular}

Note: $\sim$ Male/female represented as frequencies with the result of Chi-squared test presented. ${ }^{\$}$ Although these data are presented, there were several missing values so they were not used in further analysis.

Abbreviations: $\mathrm{BMI}$, body mass index; RV/TLC\%, ratio of residual volume to total lung volume percentage predicted; QMVC, quadriceps maximal voluntary contraction; AG, Actigraph GT3X monitor; MM, Dynaport MiniMod monitor; mvpa, moderate to vigorous physical activity; VMU, vector magnitude units; In, natural logarithm; ${ }^{\circ} \mathrm{C}$, degrees centigrade; HAD A, Hospital Anxiety and Depression Scale, Anxiety score; HAD D, Hospital Anxiety and Depression Scale, Depression score; CAT, COPD Assessment Tool; CCQ, Clinical COPD Questionnaire.

Table 2 Change of physical activity measures and 6MWD with time for patients who completed follow-up, in univariate analysis

\begin{tabular}{|c|c|c|c|c|}
\hline Variable & Baseline & 6 months & 12 months & $p$-Value ${ }^{\wedge}$ \\
\hline FEV (\% predicted) & $57.7 \pm 21.9$ & $57.2 \pm 22.6$ & $57.8 \pm 22.2$ & 0.983 \\
\hline 6MWD (m) & $449.8 \pm 127.2$ & $430.8 \pm 132.3$ & $440.5 \pm|3| .8$ & 0.839 \\
\hline AG steps & $4284(3533)$ & $3594(3212)$ & $3533(2930)$ & $<0.001$ \\
\hline AG mvpa & $8.8(18.8)$ & $7.4(I 7.4)$ & $6.1(15.7)$ & $<0.001$ \\
\hline AG VMU & $374,902.4(265,269)$ & $330,420(223,152)$ & $336,240(2 \mid 4,432)$ & $<0.001$ \\
\hline MM steps & 4690 (3708) & 4264 (3378) & 4359 (3425) & 0.001 \\
\hline MM walktime (mins) & $59.1(34.9)$ & $53.2(34.4)$ & $56.9(38.7)$ & 0.001 \\
\hline MM intensity (g) & $0.183(0)$ & $0.183(0)$ & $0.181(0)$ & $<0.001$ \\
\hline MM VMU & $286,039.6(237,72 I)$ & $265,253.2(218,109)$ & $259,447.4(199,472)$ & $<0.001$ \\
\hline
\end{tabular}

Note: ${ }^{\wedge} p$-Value for comparisons between baseline and 12-month follow-up.

Abbreviations: AG, Actigraph GT3X monitor; mvpa, moderate to vigorous physical activity; MM, Dynaport MiniMod monitor; VMU, vector magnitude units.

750 steps (Actigraph) after 1 year. Intensity, on the other hand, was found to decline significantly at 12 months follow-up, but it was similar, compared to baseline, at the 6 months follow-up. For all activity measures, recorded by both activity monitors, univariate comparison between baseline, 6 months follow-up and 12 
months follow-up, indicated that change in PA levels with time was similar across centers $(p>0.05)$, besides the differences that had been noted at baseline (data not shown). Over the course of follow-up, RV/TLC \% predicted increased significantly (baseline: $50.82 \pm 11.43$ vs 6 months: $58.79 \pm 22.92$ vs 12 months: 59.62 \pm 23.63 ; $p<0.001)$, while the rest of parameters $\left(\mathrm{FEV}_{1} \%\right.$ predicted, QMVC and CAT score) remained unchanged, compared to baseline. 6MWD was also unchanged over the 12-month period.

In multivariable analysis, time remained an independent predictor for most PA measurements, but its effect was significant only when baseline PA levels were compared to those at 12 months follow-up. The effect of time was significant on steps, mvpa and VMU (measured by both Actigraph and MiniMod monitors) (Tables 3 and 4). For instance, Actigraph stepcount was expected to be $16.9 \%$ higher when assessed at baseline, compared to 12 months follow-up (independently of age, climate conditions and changes in $\mathrm{FEV}_{1} \%$ predicted, $\mathrm{RV} / \mathrm{TLC} \%$ predicted and 6MWT). Similarly mvpa values were 1.608 times as high when assessed at baseline compared to 12 months followup. On the other hand, levels of intensity and walktime were the only two variables which were not independently associated with time in multivariable analysis.

\section{Effect of climate conditions on PA levels}

The effect of climate conditions was overall minimal and measure-specific. Rainfall ( $<1.7 \mathrm{hrs} /$ day and 1.7-2.6 hrs/ day, compared to $>2.6 \mathrm{hrs}$ /day) was negatively associated with average number of steps, as measured by both Actigraph $(\mathrm{B}=-0.205$ and -0.291 , correspondingly) and MiniMod $(\mathrm{B}=-0.258$ and -0.354 , correspondingly). For example, presence of rainfall for up to $1.7 \mathrm{hrs} /$ daily was associated to $18.5 \%$ decrease in average Actigraph step count, while raining for up to $2.6 \mathrm{hrs} /$ daily was associated to $25.2 \%$ decrease in number of steps, as measured by the same monitor. Rainfall was also negatively associated with walktime $(\mathrm{B}=-0.227$ for up to $1.7 \mathrm{hrs} /$ daily, and $\mathrm{B}=-0.309$ for raining between 1.7 and $2.6 \mathrm{hrs}$ /daily). As for VMU, a definite negative association was established between rainfall and MiniMod measurements, but for Actigraph monitor this association only tended to be statistically significant. On the contrary, rainfall had no impact on mvpa and walk intensity (Tables 3 and 4).

As for the rest of climate factors, temperature established only a negative, minimal effect on mvpa, as $1^{\circ} \mathrm{C}$ increase of average temperature was associated with approximately $3.7 \%$ decrease in mvpa measurements, while it had no impact on other PA variables. Likewise, day length had no significant impact on any PA variable measured by either Actigraph or MiniMod monitors (Tables 3 and 4) and neither did any interactions between temperature, day length and rainfall (data not shown). The latter are not presented in the final model, since their inclusion did not improve the model fit.

\section{Difference of PA levels across study sites}

The effect of recruitment site on PA measures was variable. The most profound, positive effect was established on mvpa. Athens was the study site with the strongest impact, with mvpa values being higher by more than 4.7 times, while Edinburgh was the one with the smallest impact, with mvpa values being higher by more than 1.5 times, compared to reference group. As for walktime and intensity, London was the only study site which established a minimal but significant positive (for intensity) and negative (for walktime) association with these PA measures, while no effect of any study site was established on steps or VMU measurements (Tables 3 and 4).

\section{Population subanalysis}

Two post-hoc subanalyses were conducted to test the effect of time, climate conditions and study site on each PA measure in the patient population who completed: 1) only the baseline and the 6 months follow-up (N3=184), and 2) only the baseline and the 12 months follow-up $(\mathrm{N} 4=168)$ (data not shown). In the first analysis, time was not independently associated with any PA measure, apart for mvpa $(\mathrm{B}=0.458$ for baseline visit vs 6 months follow-up; $p=0.008)$. The effect of climate conditions remained minimal, while study site had an overall significant effect only on mvpa. In the second analysis, time established an independent negative effect on all measures of PA, apart from walktime and intensity. Moreover, rainfall was again the most important of climate variables, posing a significant negative effect on most PA measures, while the effect of other ambient factors was minimal.

\section{Dropouts}

From the 236 patients who were initially recruited, only 157 patients had data for at least one activity monitor for all visits, representing a $33 \%$ dropout rate over 1 year across the whole studies (Figure 1). Overall, the subjects who dropped out of the study at some point throughout the 12 months follow-up period had lower baseline PA levels 
Table 3 Estimated coefficients and corresponding confidence intervals of GLM regressors, calculated for each physical activity parameter recorded by Dynaport MiniMod monitor

\begin{tabular}{|c|c|c|c|c|c|}
\hline PA measures & Predictor & B & Std error & $95 \% \mathrm{Cl}$ & $p$-value \\
\hline AG steps & $\begin{array}{l}\text { Visit } \\
\text {-baseline } \\
-6 \text { months follow-up } \\
-12 \text { months follow-up } \\
\text { Center } \\
\text {-Athens } \\
\text {-Edinburgh } \\
\text {-Leuven } \\
\text {-London } \\
\text {-Groningen } \\
\text { Age } \\
\text { FEVI\% predicted } \\
\text { RV/TLC\% predicted } \\
\text { QMVC } \\
6 \text { MWD } \\
\text { Temperature } \\
\text { Daylength (hrs) } \\
-<7.8 \\
-20.2 \\
->12.4 \\
\text { Rainfall (mm/day) } \\
-<1.7 \\
-4.3 \\
->2.6\end{array}$ & $\begin{array}{l}0.156 \\
0.056 \\
\text { Reference group } \\
0.172 \\
-0.229 \\
-0.067 \\
-0.066 \\
\text { Reference group } \\
-0.014 \\
0.007 \\
0.003 \\
-1.00 \mathrm{E}-4 \\
0.003 \\
0.009 \\
0.107 \\
-0.002 \\
\text { Reference group } \\
-0.205 \\
-0.291 \\
\text { Reference group }\end{array}$ & $\begin{array}{l}0.057 \\
0.074 \\
\text { Reference group } \\
0.336 \\
0.104 \\
0.077 \\
0.086 \\
\text { Reference group } \\
0.003 \\
0.001 \\
0.002 \\
0.002 \\
2.00 \mathrm{E}-04 \\
0.006 \\
0.352 \\
0.073 \\
\text { Reference group } \\
0.102 \\
0.124 \\
\text { Reference group }\end{array}$ & $\begin{array}{l}0.045-0.267 \\
-0.088-0.20 \text { I } \\
\text { Reference group } \\
-0.031-0.375 \\
-0.454 \\
-0.218-0.084 \\
-0.233-0.102 \\
\text { Reference group } \\
-0.012 \\
0.005-0.010 \\
-3 E-4-0.007 \\
-0.004-0.005 \\
0.002-0.003 \\
0.003-0.031 \\
-1.378 \\
-0.285 \\
\text { Reference group } \\
-0.401 \\
-0.487 \\
\text { Reference group }\end{array}$ & $\begin{array}{l}0.006 \\
0.446 \\
\text { Reference group } \\
0.097 \\
0.048 \\
0.387 \\
0.443 \\
\text { Reference group } \\
<0.001 \\
<0.001 \\
0.095 \\
0.964 \\
<0.001 \\
0.132 \\
0.761 \\
0.977 \\
\text { Reference group } \\
0.046 \\
0.019 \\
\text { Reference group }\end{array}$ \\
\hline AGmvpa & $\begin{array}{l}\text { Visit } \\
\text {-baseline } \\
-6 \text { months follow-up } \\
-12 \text { months follow-up } \\
\text { Centre } \\
\text {-Athens } \\
\text {-Edinburgh } \\
\text {-Leuven } \\
\text {-London } \\
\text {-Groningen } \\
\text { Age } \\
\text { FEVI\% predicted } \\
\text { RV/TLC\% predicted } \\
\text { QMVC } \\
6 \text { MWD } \\
\text { Temperature } \\
\text { Daylength (hrs) } \\
-<7.8 \\
-20.2 \\
->12.4 \\
\text { Rainfall (hrs/day) } \\
-<1.7 \\
-4.3 \\
->2.6\end{array}$ & $\begin{array}{l}0.475 \\
0.021 \\
\text { Reference group } \\
1.542 \\
0.455 \\
0.937 \\
0.874 \\
\text { Reference group } \\
-0.048 \\
0.008 \\
-0.001 \\
-0.004 \\
0.004 \\
-0.038 \\
-0.938 \\
-0.282 \\
\text { Reference group } \\
0.009 \\
-0.178 \\
\text { Reference group }\end{array}$ & $\begin{array}{l}0.141 \\
0.168 \\
\text { Reference group } \\
0.261 \\
0.288 \\
0.18 \\
0.2 \\
\text { Reference group } \\
0.007 \\
0.003 \\
0.004 \\
0.006 \\
0.001 \\
0.014 \\
0.809 \\
0.158 \\
\text { Reference group } \\
0.237 \\
0.297 \\
\text { Reference group }\end{array}$ & $\begin{array}{l}0.197-0.752 \\
-0.307-0.350 \\
\text { Reference group } \\
1.031-2.053 \\
-0.110-1.020 \\
0.584-1.291 \\
0.477-1.272 \\
\text { Reference group } \\
-0.029 \\
0.003-0.014 \\
-0.009-0.007 \\
-0.016-0.009 \\
0.003-0.005 \\
-0.056 \\
-3.172 \\
-0.62 \\
\text { Reference group } \\
-0.93 \\
-1.164 \\
\text { Reference group }\end{array}$ & $\begin{array}{l}0.001 \\
0.898 \\
\text { Reference group } \\
<0.001 \\
0.114 \\
<0.001 \\
<0.00 \text { I } \\
\text { Reference group } \\
<0.00 \text { I } \\
0.003 \\
0.847 \\
0.535 \\
<0.001 \\
0.007 \\
0.246 \\
0.075 \\
\text { Reference group } \\
0.97 \\
0.549 \\
\text { Reference group }\end{array}$ \\
\hline
\end{tabular}

(Continued) 
Table 3 (Continued).

\begin{tabular}{|c|c|c|c|c|c|}
\hline PA measures & Predictor & B & Std error & $95 \% \mathrm{Cl}$ & $p$-value \\
\hline AGVMU & $\begin{array}{l}\text { Visit } \\
\text {-baseline } \\
-6 \text { months follow-up } \\
-12 \text { months follow-up } \\
\text { Center } \\
\text {-Athens } \\
\text {-Edinburgh } \\
\text {-Leuven } \\
\text {-London } \\
\text {-Groningen } \\
\text { Age } \\
\text { FEVI\% predicted } \\
\text { RV/TLC\% predicted } \\
\text { QMVC } \\
6 \text { MWD } \\
\text { Temperature } \\
\text { Daylength (hrs) } \\
-<7.8 \\
-20.2 \\
->12.4 \\
\text { Rainfall (hrs/day) } \\
-<1.7 \\
-4.3 \\
->2.6\end{array}$ & $\begin{array}{l}0.156 \\
0.057 \\
\text { Reference group } \\
0.022 \\
-0.052 \\
-0.026 \\
-0.058 \\
\text { Reference group } \\
-0.012 \\
0.006 \\
0.001 \\
-0.003 \\
0.002 \\
0.007 \\
-0.138 \\
0.002 \\
\text { Reference group } \\
\\
-0.163 \\
-0.186 \\
\text { Reference group }\end{array}$ & $\begin{array}{l}0.046 \\
0.06 \\
\text { Reference group } \\
\\
0.083 \\
0.094 \\
0.062 \\
0.069 \\
\text { Reference group } \\
0.003 \\
0.001 \\
0.002 \\
0.002 \\
2.00 \mathrm{E}-04 \\
0.005 \\
0.287 \\
0.059 \\
\text { Reference group } \\
0.084 \\
0.101 \\
\text { Reference group }\end{array}$ & $\begin{array}{l}0.064-0.246 \\
-0.059-0.174 \\
\text { Reference group } \\
-0.141-0.184 \\
-0.237-0.133 \\
-0.148-0.097 \\
-0.193-0.076 \\
\text { Reference group } \\
-0.01 \\
0.004-0.008 \\
-0.002-0.004 \\
-0.007-0.001 \\
0.001-0.002 \\
-0.002-0.017 \\
-1.124 \\
-0.229 \\
\text { Reference group } \\
-0.329 \\
-0.397 \\
\text { Reference group }\end{array}$ & $\begin{array}{l}0.001 \\
0.335 \\
\text { Reference group } \\
0.795 \\
0.581 \\
0.68 \\
0.394 \\
\text { Reference group } \\
<0.001 \\
<0.001 \\
0.364 \\
0.154 \\
<0.001 \\
0.119 \\
0.631 \\
0.967 \\
\text { Reference group } \\
0.051 \\
0.067 \\
\text { Reference group }\end{array}$ \\
\hline
\end{tabular}

Abbreviations: RV/TLC\%, ratio of residual volume to total lung volume percentage predicted; GLM, generalized linear model; PA, physical activity; Std, standard; AG, Actigraph GT3X monitor; RV, residual volume; TLC, total lung capacity; QMVC, quadriceps maximal voluntary contraction; mvpa, moderate to vigorous physical activity; VMU, vector magnitude units; E-4, $10^{-4}$.

(step count, mvpa, VMU, PA intensity, walktime) as measured by either activity monitor $(p<0.05)$. They also had a lower baseline 6MWD ( $p=0.001)$, lower QMVC $(p=0.003)$ and worse quality of life, as suggested by the higher CCQ scores $(p=0.032$ ), compared to those who did not dropout. No difference was noted in exacerbation frequency between the groups $(p=0.67)$ and the groups were well matched for age, BMI, $\mathrm{FEV}_{1} \%$ predicted and RV/TLC\% predicted. Weather conditions at baseline were also similar, apart from temperature which was approximately $2^{\circ} \mathrm{C}$ lower in the group of patients who dropped out.

\section{Discussion}

In this multicenter, prospective, cohort study ${ }^{18}$ we investigated the effect of ambient climate factors on the progression of PA in COPD as measured by two validated, commercially available PA monitors, at baseline, 6 months and 12 months. There was a significant effect of time with a decline in almost every measure of physical activity over 12 months. The number of hours with rainfall daily had a negative effect on most physical activity variables, but otherwise analysis indicated hardly any associations between PA and ambient conditions. The effect of study site was most evident on mvpa, while for the rest of PA measures it was variable and inconsistent.

\section{Significance of findings}

The most important outcome of the study is establishing that the passage of time itself is the most significant predictor of PA decline over 12 months, as indicated by most Actigraph and MiniMod monitor measures, especially given the importance of physical activity as the strongest predictor of all-cause mortality in COPD. ${ }^{29}$ Previous studies suggested that PA declines with time among COPD patients, ${ }^{8,9}$ however, these were single-center studies, with the follow-up period being much longer. In our study, the decline of PA applied to all centers, despite the patients having (by design) a wide range in baseline characteristics, including age, $\mathrm{FEV}_{1}$, climate conditions, 6MWD and PA and, thus, this is a finding with positive implications for 
Table 4 Estimated coefficients and corresponding confidence intervals of GLM regressors, calculated for each physical activity parameter recorded by Dynaport MiniMod monitor

\begin{tabular}{|c|c|c|c|c|c|}
\hline PA measures & Predictor & B coefficients & Std error & $95 \% \mathrm{Cl}$ & $p$-value \\
\hline MM steps & $\begin{array}{l}\text { Visit } \\
\text {-baseline } \\
-6 \text { months follow-up } \\
-12 \text { months follow-up } \\
\text { Center } \\
\text {-Athens } \\
\text {-Edinburgh } \\
\text {-Leuven } \\
\text {-London } \\
\text {-Groningen } \\
\text { Age } \\
\text { FEVI\% predicted } \\
\text { RV/TLC\% predicted } \\
\text { QMVC } \\
6 \text { MWD } \\
\text { Temperature } \\
\text { Daylength (hrs) } \\
-<7.8 \\
-20.2 \\
->12.4 \\
\text { Rainfall (hrs/day) } \\
-<1.7 \\
-4.3 \\
->2.6\end{array}$ & $\begin{array}{l}0.029 \\
-0.08 \\
\text { Reference group } \\
0.127 \\
-0.237 \\
-0.054 \\
-0.156 \\
\text { Reference group } \\
-0.006 \\
0.006 \\
0.003 \\
-2.10 \mathrm{E}-4 \\
0.003 \\
0.006 \\
-0.051 \\
-0.091 \\
\text { Reference group } \\
-0.258 \\
-0.354 \\
\text { Reference group }\end{array}$ & $\begin{array}{l}0.059 \\
0.077 \\
\text { Reference group } \\
0.107 \\
0.124 \\
0.081 \\
0.093 \\
\text { Reference group } \\
0.003 \\
0.001 \\
0.002 \\
0.003 \\
3.00 \mathrm{E}-4 \\
0.006 \\
0.354 \\
0.076 \\
\text { Reference group } \\
0.105 \\
0.128 \\
\text { Reference group }\end{array}$ & $\begin{array}{l}0.086-0.145 \\
-0.232-0.072 \\
\text { Reference group } \\
-0.081-0.336 \\
-0.479-0.005 \\
-0.212-0.104 \\
-0.337-0.026 \\
\text { Reference group } \\
-0.013-4.4 \mathrm{E}-5 \\
0.004-0.009 \\
-0.001-0.007 \\
-0.005-0.005 \\
0.002-0.003 \\
-0.006-0.018 \\
-0.746-0.643 \\
-0.240-0.058 \\
\text { Reference group } \\
-0.412 \\
-0.5 \\
\text { Reference group }\end{array}$ & $\begin{array}{l}0.033 \\
0.301 \\
\text { Reference group } \\
0.232 \\
0.055 \\
0.503 \\
0.093 \\
\text { Reference group } \\
0.048 \\
<0.001 \\
0.11 \\
0.932 \\
<0.001 \\
0.313 \\
0.885 \\
0.23 \\
\text { Reference group } \\
0.014 \\
0.006 \\
\text { Reference group }\end{array}$ \\
\hline MM walktime & $\begin{array}{l}\text { Visit } \\
\text {-baseline } \\
-6 \text { months follow-up } \\
-12 \text { months follow-up } \\
\text { Center } \\
\text {-Athens } \\
\text {-Edinburgh } \\
\text {-Leuven } \\
\text {-London } \\
\text {-Groningen } \\
\text { Age } \\
\text { FEVI\% predicted } \\
\text { RV/TLC\% predicted } \\
\text { QMVC } \\
6 \text { MWD } \\
\text { Temperature } \\
\text { Daylength (hrs) } \\
-<7.8 \\
-20.2 \\
->12.4 \\
\text { Rainfall (hrs/day) } \\
-<1.7 \\
-4.3 \\
->2.6\end{array}$ & $\begin{array}{l}0.026 \\
-0.09 \\
\text { Reference group } \\
0.107 \\
-0.214 \\
-0.042 \\
-0.169 \\
\text { Reference group } \\
-0.005 \\
0.005 \\
0.003 \\
0.002 \\
0.002 \\
0.006 \\
-0.027 \\
-0.098 \\
\text { Reference group } \\
-0.227 \\
-0.309 \\
\text { Reference group }\end{array}$ & $\begin{array}{l}0.055 \\
0.072 \\
\text { Reference group } \\
0.099 \\
0.115 \\
0.075 \\
0.085 \\
\text { Reference group } \\
0.003 \\
0.001 \\
0.002 \\
0.002 \\
2.00 \mathrm{E}-4 \\
0.006 \\
0.328 \\
0.07 \\
\text { Reference group } \\
0.097 \\
0.118 \\
\text { Reference group }\end{array}$ & $\begin{array}{l}-0.081-0.133 \\
-0.231-0.05 \text { I } \\
\text { Reference group } \\
-0.440-0.011 \\
-0.188-0.104 \\
-0.334 \\
-0.082-0.133 \\
\text { Reference group } \\
-0.0109 \\
0.003-0.008 \\
-0.001-0.006 \\
-0.003-0.067 \\
0.002-0.003 \\
-0.005-0.016 \\
-0.669-0.615 \\
-0.235-0.040 \\
\text { Reference group } \\
-0.382 \\
-0.462 \\
\text { Reference group }\end{array}$ & $\begin{array}{l}0.632 \\
0.21 \\
\text { Reference group } \\
0.278 \\
0.062 \\
0.57 \\
0.048 \\
\text { Reference group } \\
0.048 \\
<0.001 \\
0.147 \\
0.396 \\
<0.001 \\
0.312 \\
0.934 \\
0.165 \\
\text { Reference group } \\
0.02 \\
0.009 \\
\text { Reference group }\end{array}$ \\
\hline
\end{tabular}

(Continued) 
Table 4 (Continued).

\begin{tabular}{|c|c|c|c|c|c|}
\hline PA measures & Predictor & B coefficients & Std error & $95 \% \mathrm{Cl}$ & $p$-value \\
\hline MM intensity & $\begin{array}{l}\text { Visit } \\
\text {-baseline } \\
-6 \text { months follow-up } \\
-12 \text { months follow-up } \\
\text { Center } \\
\text {-Athens } \\
\text {-Edinburgh } \\
\text {-Leuven } \\
\text {-London } \\
\text {-Groningen } \\
\text { Age } \\
\text { FEVI\% predicted } \\
\text { RV/TLC\% predicted } \\
\text { QMVC } \\
6 \text { MWD } \\
\text { Temperature } \\
\text { Daylength (hrs) } \\
-<7.8 \\
-20.2 \\
->12.4 \\
\text { Rainfall (hrs/day) } \\
-<1.7 \\
-4.3 \\
->2.6\end{array}$ & $\begin{array}{l}0.015 \\
0.009 \\
\text { Reference group } \\
0.043 \\
0.035 \\
-0.027 \\
0.081 \\
\text { Reference group } \\
-0.006 \\
0.002 \\
-3.50 \mathrm{E}-4 \\
-0.001 \\
4.80 \mathrm{E}-4 \\
-7.50 \mathrm{E}-5 \\
0.043 \\
-0.004 \\
\text { Reference group } \\
-0.012 \\
-0.035 \\
\text { Reference group }\end{array}$ & $\begin{array}{l}0.015 \\
0.02 \\
\text { Reference group } \\
0.027 \\
0.033 \\
0.021 \\
0.024 \\
\text { Reference group } \\
8.00 \mathrm{E}-4 \\
3.00 \mathrm{E}-4 \\
5.00 \mathrm{E}-4 \\
6.00 \mathrm{E}-4 \\
6.50 \mathrm{E}-5 \\
0.002 \\
0.092 \\
0.019 \\
\text { Reference group } \\
0.027 \\
0.033 \\
\text { Reference group }\end{array}$ & $\begin{array}{l}-0.015-0.045 \\
-0.030-0.048 \\
\text { Reference group } \\
-0.011-0.097 \\
-0.028-0.099 \\
-0.067-0.014 \\
0.035-0.127 \\
\text { Reference group } \\
-0.003 \\
0.001-0.002 \\
-0.001-0.001 \\
-0.002914 \\
3.6 E-4-0.001 \\
-0.003-0.003 \\
-0.137-0.222 \\
-0.042-0.033 \\
\text { Reference group } \\
-0.065-0.042 \\
-0.100-0.029 \\
\text { Reference group }\end{array}$ & $\begin{array}{l}0.333 \\
0.648 \\
\text { Reference group } \\
0.118 \\
0.279 \\
0.198 \\
0.001 \\
\text { Reference group } \\
<0.001 \\
<0.001 \\
0.476 \\
0.036 \\
<0.001 \\
0.961 \\
0.642 \\
0.817 \\
\text { Reference group } \\
0.668 \\
0.284 \\
\text { Reference group }\end{array}$ \\
\hline MMVMU & $\begin{array}{l}\text { Visit } \\
\text {-baseline } \\
-6 \text { months follow-up } \\
-12 \text { months follow-up } \\
\text { Center } \\
\text {-Athens } \\
\text {-Edinburgh } \\
\text {-Leuven } \\
\text {-London } \\
\text {-Groningen } \\
\text { Age } \\
\text { FEVI\% predicted } \\
\text { RV/TLC\% predicted } \\
\text { QMVC } \\
6 \text { MWD } \\
\text { Temperature } \\
\text { Daylength (hrs) } \\
-<7.8 \\
-20.2 \\
->12.4 \\
\text { Rainfall (hrs/day) } \\
-<1.7 \\
-4.3 \\
->2.6\end{array}$ & $\begin{array}{l}0.085 \\
-0.01 \\
\text { Reference group } \\
0.129 \\
-0.065 \\
-0.114 \\
0.002 \\
\text { Reference group } \\
-0.016 \\
0.008 \\
0.001 \\
-0.005 \\
0.003 \\
0.001 \\
-0.258 \\
-0.066 \\
\text { Reference group } \\
-0.238 \\
-0.339 \\
\text { Reference group }\end{array}$ & $\begin{array}{l}0.056 \\
0.072 \\
\text { Reference group } \\
0.1 \\
0.116 \\
0.076 \\
0.087 \\
\text { Reference group } \\
0.003 \\
0.001 \\
0.002 \\
0.002 \\
2.00 \mathrm{E}-4 \\
0.006 \\
0.332 \\
0.07 \\
\text { Reference group } \\
0.098 \\
0.119 \\
\text { Reference group }\end{array}$ & $\begin{array}{l}0.024-0.194 \\
-0.152-0.131 \\
\text { Reference group } \\
-0.067-0.325 \\
-0.293-0.163 \\
-0.263-0.035 \\
-0.168-0.172 \\
\text { Reference group } \\
-0.012 \\
0.005-0.010 \\
-0.003-0.004 \\
-0.00951 \\
0.002-0.003 \\
-0.010-0.012 \\
-0.909-0.394 \\
-0.203-0.070 \\
\text { Reference group } \\
-0.388 \\
-0.468 \\
\text { Reference group }\end{array}$ & $\begin{array}{l}0.026 \\
0.885 \\
\text { Reference group } \\
0.197 \\
0.576 \\
0.133 \\
0.983 \\
\text { Reference group } \\
<0.001 \\
<0.001 \\
0.699 \\
0.03 \\
<0.001 \\
0.83 \\
0.438 \\
0.342 \\
\text { Reference group } \\
0.016 \\
0.005 \\
\text { Reference group }\end{array}$ \\
\hline
\end{tabular}

Abbreviations: QMVC, quadriceps maximal voluntary contraction; RV/TLC\%, ratio of residual volume to total lung volume percentage predicted; E-4, $10^{-4}$; E-5, $10^{-5}$. 
future studies in COPD patients, suggesting that PA can be used as an outcome measure over periods as short as 1 year. Surprisingly two PA measures, namely walktime and intensity, although significantly declining over the 12-month period, were not significantly associated with time per se in multivariate analysis. This may be because 12 months are not long enough for elapsed time to establish its independent effect on these two measures, but this is a hypothesis that will need further investigation. In any case, establishing which PA measures decline over time provides guidance for early interventions to preserve physical activity levels among COPD patients and for the design of studies to evaluate interventions to enhance or preserve physical activity.

The effect of climate factors on PA was measure-specific overall. Rainfall hours were the main climate variable which posed a significant negative effect on average number of steps, walktime and VMU, and this effect was only evident over the 12 months follow-up. These findings are consistent with a previous study where high levels of rain (more than 10 $\mathrm{mm} /$ daily) resulted to decreased average number of steps daily, ${ }^{30}$ among COPD individuals. Some previously published, single-center studies indicated that daily temperature might also be a significant predictor of daily PA, with higher temperature levels being associated with greater PA. ${ }^{14,15,30}$ In our study, however, temperature established only a minimal, negative effect on mvpa, a result that is difficult to explain, since this association has not been assessed before. During the 12 months follow-up period, the recorded temperatures in different study sites ranged from $-4.5^{\circ} \mathrm{C}$ (in Leuven) to approximately $38^{\circ} \mathrm{C}$ (in Athens), so the lack of an impact of temperature on several PA measures cannot be attributed to the narrow range of temperature values included in the analysis. It is likely that our use of a weekly average may have masked the potential effects of temperature on PA at the daily level. Furthermore, no effect was evident for day length, nor its interactions with any other climate variable. Currently, the published studies ${ }^{14,15,31}$ looking at the effect of climate on PA in COPD are few and they are not without limitations. In the most recent study, Alahmari et al investigated the effect of weather data (temperature, rainfall, sunshine), environmental particulate matter $<10 \mu \mathrm{m}\left(\mathrm{PM}_{10}\right)$ and ozone levels on PA using pedometers, in a cohort of COPD patients who were followed up daily. The authors found step count to be lower on colder and overcast days, while there was also a negative impact of atmospheric $\mathrm{PM}_{10}$ and ozone levels, on step count. ${ }^{14}$ However, this study involved a smaller number of patients, with a very wide variation in follow- up period ranging from 1 month to almost 21 months, while results were not adjusted for age, $\mathrm{FEV}_{1} \%$ predicted and other disease severity measures. In the study of Moy et al, where season was found to have some effect on step count, the cohort was made up mostly of male patients, the follow-up period was short, while there were no objective measurements regarding climatic conditions, such as temperature or sunshine, to define the seasons. ${ }^{15}$ Maximum temperature, sunshine and day length were positive independent predictors of daily PA in another study among elderly individuals; nevertheless, these were subjects without COPD, while the PA and climate data were recorded for a very short period. ${ }^{31}$ In the most similar study in the filed, minimum and maximum temperature, daylight duration and humidity had some impact on PA among COPD patients; nevertheless, this study included a small patient population, while duration of followup was shorter and temperature ranges smaller. ${ }^{32}$ In conclusion, the significant effect of rainfall, but not of any other climate condition, on several measures of PA levels in our study, after controlling for elapsed time and other major determinants of disease severity among COPD patients, indicate that climate factors are probably not major determinants of PA levels in this population.

To the authors' knowledge, the current study is the only published one that investigated the effect of climate conditions on several PA parameters, measured using two different activity monitors worn simultaneously by patients. In other similar studies in the field, the main measure used was step count. ${ }^{14,15,30}$ Although the accuracy of both monitors (Actigraph and MiniMod) in measuring PA among COPD patients is similar and has been previously confirmed, ${ }^{25,26}$ it might be the case that some measures of PA may be less sensitive in detecting any impact of climate conditions than others, especially since this effect seems to be present, but not very profound. However, this is an assumption that needs to be further investigated.

The effect of study site on PA levels was variable and the interpretation of this result lies probably beyond disease severity. The PA measure mostly affected by study site was mvpa, while for step count and VMU, no effect of study site was observed. Previously published data indicate that several socio-cultural, socio-economic and environmental factors, which could vary significantly in different communities, may determine the level of PA in adults. $^{33,34}$ Micro- and macroenvironment in the area of living seems to be an important determinant of PA with high street connectivity, high neighborhood walkability, 
availability and proximity of transport system being positively associated with daily PA. Likewise, adverse street characteristics, such as lack of sidewalks and streetlights are negatively associated with PA among adults. ${ }^{35,34}$ Unfortunately, these environmental and neighborhood data were not available in our study, so they were not controlled for; however, since they could cause some differences between sites, selecting PA variables that seem to be less affected by study site, such as step count or VMU as outcome measures, could possible decrease diversity in studies with multicenter protocol. Nevertheless, even if these site differences exist at baseline, they are unlikely to change over the time course of a clinical trial.

Another interesting observation was that baseline temperature was approximately $2^{\circ} \mathrm{C}$ lower in the group of patients that dropped out of the study. These patients also had lower baseline PA, as established by both activity monitors and lower exercise capacity, lower QMVC and higher CCQ scores, compared to the ones who completed the study. These characteristics mirror the findings in pulmonary rehabilitation studies where those patients who drop out have worse exercise capacity, are more breathless, have poorer health-related quality of life and weaker quadriceps strength. ${ }^{37,36}$ Whether these patients might also be more sensitive to the effect of environmental conditions is yet to be investigated.

\section{Methodological issues}

The current study has several strengths, but also carries some limitations, as expected. It is a part of a large, multicenter project, conducted in different geographical sites, which collected a large amount of PA and climate data using two previously validated activity monitors, over three time points, among COPD patients with a wide range of disease severity. ${ }^{18}$ Data were all centrally collected and checked, based on predefined rules, in order to minimize site variability due to potential methodological bias. Unfortunately, the analysis of climate was limited by the availability of data, since number of hours of sunshine per day was not available for all centers, and other factors such as humidity or pollution levels were not available for any site. Furthermore, severity of rainfall, measured in $\mathrm{mm}$ per hour was not analyzed, as these data were not available for every site. Another limitation is the use of weekly averages of climate variables, which may have decreased the daily effect of climate variation on PA. Moreover, the season of patient recruitment was not analyzed, since not only in some centers the seasonal differences in climate are less profound compared to other centers, but also in real life there is a gradual transition from one season to the next, so grouping patients by season would be arbitrary; the interaction of temperature with day length was used instead, as an objective surrogate of seasonal effect, although it remained a non-significant factor in statistical analysis. Finally, it is unfortunate but expected that patients dropped out of the study after their initial or 6month visit. In the single-center study of Watchki et al , the dropout rate was $20 \%,{ }^{9}$ which is lower than the present study (33\%), but still a significant number, while in similar studies conducted among COPD patients who undergo pulmonary rehabilitation, dropout rates may be as high as $30-40 \%{ }^{37,36}$ In any case this is a finding that has to be accounted for in future studies, especially in sample size calculations.

\section{Conclusion}

We have demonstrated a decline in PA in people with COPD over 1-year follow-up, with elapsed time itself being an independent predictor of this change, in the absence of significant decline in $\mathrm{FEV}_{1}$ or $6 \mathrm{MWD}$. If PA declines more consistently than other clinically important measures in COPD population, such as exercise capacity or lung function, it may represent a more sensitive outcome measure for use when designing future studies in COPD. Overall, the effect of climate on PA decline was inconsistent, although more hours with rainfall during the day were associated with lower PA and cooler temperatures seemed to be associated with an increased dropout rate. Since the background effects of time on PA over 6 months are fairly small, we can conclude that a 6 months follow-up period for a trial would be reasonable, without the need to require 12 months, so that climate conditions are broadly matched between baseline and study end. In this case mvpa is probably a less appropriate PA variable to measure, since it is the only one affected by elapsed time, ambient conditions and study site, during follow-up periods shorter than a year. These findings should be useful in the design of future multicenter studies using PA as an outcome measure.

\section{Acknowledgment}

This study is supported EU Innovative Medicines Initiative Joint Undertaking (IMU JU) \#115011. 


\section{Author contributions}

All authors contributed to data analysis, drafting or revising the article, gave final approval of the version to be published, and agree to be accountable for all aspects of the work.

\section{Disclosure}

JGA received payments for consulting and lecture fees to her Institution from AstraZeneca and lecture fees from Esteeve and Chiesi, outside the submitted work. MIP received personal fees from Phillips, grants from EU and travel expenses from $\mathrm{CBQC}$, outside the submitted work. TvdM is currently a part-time employee of GSK London. The authors report no other conflicts of interest in this work.

\section{References}

1. Vestbo J, Hurd SS, Agustí AG, et al. Global strategy for the diagnosis, management, and prevention of chronic obstructive pulmonary disease: GOLD executive summary. Am J Respir Crit Care Med. 2013;187:347-365. doi:10.1164/rccm.201204-0596PP

2. Oga T, Nishimura $\mathrm{K}$, Tsukino $\mathrm{M}$, et al. Longitudinal deteriorations in patient reported outcomes in patients with COPD. Respir Med. 2007;101:146-153. doi:10.1016/j.rmed.2006.04.001

3. Van Remoortel H, Hornikx M, Demeyer H, et al. Daily physical activity in subjects with newly diagnosed COPD. Thorax. 2013;68 (10):962-963. doi:10.1136/thoraxjnl-2013-203534

4. Shrikrishna D, Patel M, Tanner RJ, et al. Quadriceps wasting and physical inactivity in patients with COPD. Eur Respir J. 2012;40 (5):1115-1122. doi:10.1183/09031936.00170111

5. Gimeno-Santos E, Frei A, Steurer-Stey C, et al.; PROactive consortium. Determinants and outcomes of physical activity in patients with COPD: a systematic review. Thorax. 2014;69(8):731-739. doi:10.1136/thoraxjnl-2013-204763.

6. Van Remoortel H, Hornikx M, Langer D, et al. Risk factors and comorbidities in the preclinical stages of chronic obstructive pulmonary disease. Am J Respir Crit Care Med. 2014;189:30-38. doi:10.1164/rccm.201306-1150OC

7. Watz H, Pitta F, Rochester CL, et al. An official European Respiratory Society statement on physical activity in COPD. Eur Respir J. 2014;44(6):1521-1537. doi:10.1183/09031936.00046814

8. Agarwal V, Tetenta S, Bautista J, et al. Longitudinal changes in directly measured physical activity in patients with chronic obstructive pulmonary disease: the trajectory of change. $J$ Cardiopulm Rehabil Prev. 2012;32(5):292-295. doi:10.1097/HCR.0b013e31825c4242

9. Waschki B, Kirsten HO, Holz O, et al. Disease progression and changes in physical activity in patients with chronic obstructive pulmonary disease. Am J Respir Crit Care Med. 2015;192(3):295306. doi:10.1164/rccm.201501-0081OC

10. Sievi NA, Brack T, Brutsche MH, et al. Physical activity declines in COPD while exercise capacity remains stable: a longitudinal study over 5 years. Respir Med. 2018;141:1-6. doi:10.1016/j.rmed.2018.06.013

11. Demeyer H, Donaire-Gonzalez D, Gimeno-Santos E, et al. Physical activity is associated with attenuated disease progression in chronic obstructive pulmonary disease. Med Sci Sports Exerc. 2019;51 (5):833-840. doi:10.1249/MSS.0000000000001859

12. Tucker P, Gilliland J. The effect of season and weather on physical activity: a systematic review. Public Health. 2007;121(12):909-922. doi:10.1016/j.puhe.2007.04.009
13. Demeyer H, Louvaris Z, Frei A, et al.; PROactive study group and the PROactive consortium. Physical activity is increased by a 12week semiautomated telecoaching programme in patients with COPD: a multicentre randomised controlled trial. Thorax. 2017;72 (5):415-423. doi:10.1136/thoraxjnl-2016-209026.

14. Alahmari AD, Mackay AJ, Patel AR, et al. Influence of weather and atmospheric pollution on physical activity in patients with COPD. Respir Res. 2015;16:71. doi:10.1186/s12931-015-0229-z

15. Moy ML, Danilack VA, Weston NA, et al. Daily step counts in a US cohort with COPD. Respir Med. 2012;106(7):962-969. doi:10.1016/j. rmed.2012.03.016

16. Sewell L, Singh SJ, Williams JE, et al. Seasonal variations affect physical activity and pulmonary rehabilitation outcomes. $J$ Cardiopulm Rehabil Prev. 2010;30(5):329-333. doi:10.1097/ HCR.0b013e3181e175f2

17. Nakao M, Ishihara Y, Kim CH, Hyun IG. The impact of air pollution, including Asian sand dust, on respiratory symptoms and healthrelated quality of life in outpatients with chronic respiratory disease in Korea: a panel study. J Prev Med Public Health. 2018;51(3):130139. doi:10.3961/jpmph.18.021

18. Gimeno-Santos E, Raste Y, Demeyer H, et al.; PROactive consortium. The PROactive instruments to measure physical activity in patients with chronic obstructive pulmonary disease. Eur Respir J. 2015;46(4):988-1000. doi:10.1183/09031936.00183014.

19. Demeyer H, Gimeno-Santos E, Rabinovich RA, et al.; PROactive consortium. Physical activity characteristics across GOLD quadrants depend on the questionnaire used. PLoS One. 2016;11(3):e0151255. doi:10.1371/journal.pone. 0151255 .

20. Dueñas-Espín I, Demeyer H, Gimeno-Santos E, et al. Depression symptoms reduce physical activity in COPD patients: a prospective multicenter study. Int J Chron Obstruct Pulmon Dis. 2016;10 (11):1287-1295. doi:10.2147/COPD.S101459

21. Jones PW, Harding G, Berry P, Wiklund I, Chen WH, Leidy NK. Development and first validation of the COPD assessment test. Eur Respir J. 2009;34:648-654. doi:10.1183/09031936.00102509

22. Zigmond AS, Snaith RP. The hospital anxiety and depression scale. Acta Psychiatr Scand. 1983;67:361-370. doi:10.1111/acp.1983.67. issue-6

23. van der Molen T, Willemse BW, Schokker S, Ten Hacken NH, Postma DS, Juniper EF. Development, validity and responsiveness of the Clinical COPD Questionnaire. Health Qual Life Outcomes. 2003;1:13. doi:10.1186/1477-7525-1-13

24. ATS. Committee on proficiency standards for clinical pulmonary function laboratories. ATS statement: guidelines for the six-minute walk test. Am J Respir Crit Care Med. 2002;166(1):111-117. doi:10.1164/ajrccm.166.1.at1102

25. Rabinovich RA, Louvaris Z, Raste Y, et al.; PROactive Consortium. Validity of physical activity monitors during daily life in patients with COPD. Eur Respir J. 2013;42(5):1205-1215. doi:10.1183/ 09031936.00134312.

26. Van Remoortel H, Raste Y, Louvaris Z, et al.; PROactive consortium. Validity of six activity monitors in chronic obstructive pulmonary disease: a comparison with indirect calorimetry. PLoS One. 2012;7 (6):e39198. doi:10.1371/journal.pone.0039198.

27. Fox J. Generalised Linear Models. In: Applied Regression Analysis and Generalized Linear Models. 3rd ed. Sage Publications; 2016:379-424.

28. Akaike H. A new look at the statistical model identification. IEEE Trans Automat Contr. 1974;19(6):716-723. doi:10.1109/TAC.1974.1100705

29. Waschki B, Kirsten A, Holz O, et al. Physical activity is the strongest predictor of all-cause mortality in patients with COPD: a prospective cohort study. Chest. 2011;140(2):331-342. doi:10.1378/chest.102521

30. Balish SM, Dechman G, Hernandez P, et al. The relationship between weather and objectively measured physical activity among individuals with COPD. J Cardiopulm Rehabil Prev. 2017;37(6):445-449. doi:10.1097/HCR.0000000000000244 
31. Sumukadas D, Witham M, Struthers A, et al. Day length and weather conditions profoundly affect physical activity levels in older functionally impaired people. J Epidemiol Community Health. 2009;63 (4):305-309. doi:10.1136/jech.2008.080838

32. Furlanetto KC, Demeyer H, Sant'anna T, et al. Physical activity of patients with COPD from regions with different climatic variations. COPD. 2017;14(3):276-283. doi:10.1080/15412555.2017.1303039

33. Jaeschke L, Steinbrecher A, Luzak A, et al.; DEDIPAC consortium. Socio-cultural determinants of physical activity across the life course: a 'Determinants of Diet and Physical Activity' (DEDIPAC) umbrella systematic literature review. Int J Behav Nutr Phys Act. 2017;14 (1):173. doi:10.1186/s12966-017-0627-3.

34. O'Donoghue G, Kennedy A, Puggina A, et al. Socio-economic determinants of physical activity across the life course: a "DEterminants of DIet and Physical ACtivity" (DEDIPAC) umbrella literature review. PLoS One. 2018;13(1):e0190737. doi:10.1371/journal.pone.0190737
35. Carlin A, Perchoux C, Puggina A, et al. A life course examination of the physical environmental determinants of physical activity behaviour: a "Determinants of Diet and Physical Activity" (DEDIPAC) umbrella systematic literature review. PLoS One. 2017;12(8): e0182083. doi:10.1371/journal.pone. 0182083

36. Hajna S, Ross NA, Brazeau AS, et al. Associations between neighbourhood walkability and daily steps in adults: a systematic review and meta-analysis. BMC Public Health. 2015;15:768. doi:10.1186/ s12889-015-2082-x

37. Garrod R, Marshall J, Barley E, et al. Predictors of success and failure in pulmonary rehabilitation. Eur Respir J. 2006;27(4):788794. doi:10.1183/09031936.06.00130605

38. Boutou AK, Tanner RJ, Lord VM, et al. An evaluation of factors associated with completion and benefit from pulmonary rehabilitation in COPD. BMJ Open Respir Res. 2014;1(1):e000051. doi:10.1136/ bmjresp-2014-000051

\section{Publish your work in this journal}

The International Journal of COPD is an international, peer-reviewed journal of therapeutics and pharmacology focusing on concise rapid reporting of clinical studies and reviews in COPD. Special focus is given to the pathophysiological processes underlying the disease, intervention programs, patient focused education, and self management protocols. This journal is indexed on PubMed Central, MedLine and CAS. The manuscript management system is completely online and includes a very quick and fair peer-review system, which is all easy to use. Visit http://www.dovepress.com/testimonials.php to read real quotes from published authors. 